\title{
Das Leck in der Lunge: Bronchoskopische Ventil- implantation zur Behandlung einer persistierenden bronchopleuralen Fistel
}

\author{
A Leak in the Lung: Endobronchial One-Way Valve Placement as Treatment \\ for a Persistent Bronchopleural Fistula
}

Autoren

Institut
U. Seyfried, I. Firlinger, M. Reiter, E. Katz-Papatheophilou, H. Koller, O. C. Burghuber, A. Valipour

I. Interne Lungenabteilung, Ludwig-Boltzmann-Institut für COPD und Pneumologische Epidemiologie, Otto-Wagner-Spital, Wien eingereicht 7.10.2011 akzeptiert nach Revision 12. 12.2011

\section{Bibliografie}

Dol http://dx.doi.org/ 10.1055/s-0031-1291615 Online-Publikation: 27.1.2012 Pneumologie 2012; 66: 188-191 (c) Georg Thieme Verlag KG Stuttgart · New York ISSN 0934-8387

Korrespondenzadresse

Priv.-Doz. Dr.

\section{Arschang Valipour}

I. Interne Lungenabteilung mit Intensivstation Ludwig-Boltzmann-Institut für COPD und Pneumologische Epidemiologie Otto-Wagner-Spital, Sanatoriumstr.2 1140 Wien arschang.valipour@wienkav.at

\section{Zusammenfassung \\ $\nabla$}

Das Vorhandensein einer bronchopleuralen Fistel ist mit einem erhöhtem Morbiditäts- und Mortalitätsrisiko verbunden. Die Therapie umfasst neben intrathorakaler Drainage und Antibiose den Fistelverschluss, welcher im Regelfall eine chirurgische Domäne darstellt. Bei Pat. mit eingeschränkter Lungenfunktion ist in vielen Fällen eine minimal-invasive Alternative wünschenswert bzw. erforderlich. Wir berichten über eine 62-jährige Pat. mit schwerer COPD, die aufgrund eines Lungenabszesses zur Aufnahme gelangte und eine bronchopleurale Fistel entwickelte. Die Behandlung umfasste neben einer antibiotischen Therapie die Implantation endobronchialer Einwegventile, welche zum Fistelverschluss und schließlich zur Rekonvaleszenz führte.

\section{Einleitung}

Bei einer bronchopleuralen Fistel (BPF) handelt es sich um eine temporäre oder persistierende pathologische Verbindung zwischen dem Bronchialsystem und dem Pleuraraum. Hinsichtlich der Genese der BPF muss in der Regel unterschieden werden, ob es sich um eine periphere Parenchymleckage (im angloamerikanischen Raum auch gerne als „Air Leak“ bezeichnet) handelt oder um eine BPF als Ausdruck einer nachweisbaren Bronchusstumpfinsuffizienz nach thorakalen Eingriffen.

Nach Baumann et al. [1] sind in zwei Drittel der Fälle ursächlich thoraxchirurgische Eingriffe (Lungenresektionen unterschiedlichen Ausmaßes und v.a. Pleurektomien) für die Entstehung der BPF verantwortlich. Das restliche Drittel geht auf Infektionen (Pleurapneumonie, Lungenabszess, Tuberkulose) bzw. in selteneren Fällen auch auf einen Spontanpneumothorax zurück [1 -3]. Prädisponierend für das Auftreten sind insbesondere vorbestehende pulmonale Parenchymer-

\section{Abstract \\ $\nabla$}

Patients with bronchopleural fistula are at an increased risk of morbidity and mortality. Treatment of the air leak includes intrathoracic drainage, antibiotic therapy and closure of the fistula, which conventionally has been performed via surgical means. In patients with limited respiratory capacity, less-invasive alternatives are required. Here we report on a 62-year-old patient with underlying severe COPD, who was admitted with a lung abscess and consecutively developed a persistent bronchopleural fistula. Treatment involved antibiotic therapy and endobronchial oneway valve placement, which resulted in termination of the air leak and full recovery.

krankungen, wie das Lungenemphysem, Lungenfibrosen, Lungenkarzinom, sowie ein schlechter Allgemeinzustand [4-8]. Das Vorhandensein einer BPF führt nachgewiesenermaßen zu einer erhöhten Morbidität und Mortalität [4,5,9,10], darüber hinaus kommt ein beträchtlicher sozioökonomischer Faktor hinzu, da meistens von einer langwierigen und kostspieligen Behandlung auszugehen ist. Wenn das primäre Vorgehen einer thoraxchirurgischen Sanierung nicht möglich ist, dann muss entsprechend des klinischen Zustandes und der funktionellen Gegebenheiten des Patienten eine individuelle Lösung gefunden werden. Bei bis dato fehlenden allgemein gültigen Leitlinien sind hierfür in der Literatur verschiedenste endoskopische Verfahren beschrieben worden; wir wollen nun die Verwendung eines endobronchialen Einwegventils bei persistierender BPF anhand eines Fallbeispiels beschreiben und diskutieren. 


\section{Fallbericht}

\section{$\nabla$}

Wir berichten über eine 62-jährige Pat., die aufgrund eines seit wenigen Wochen bestehenden, produktiven Hustens und zunehmender Belastungsdyspnoe bei vorbekannter chronisch obstruktiver Atemwegserkrankung (COPD Stadium GOLD IV) mit dem klinischen und radiologischen Bild einer Pneumonie stationär aufgenommen wurde. Bereits im Vorfeld erhielt die Patientin eine antibiotische Therapie mit einem Aminopenicillin/Betalaktamasehemmer, in Kombination mit einem Makrolidantibiotikum bzw. nachfolgend ein Fluorchinolon verabreicht. In der Anamnese ist auf einen Zustand nach Konisation und Curettement, einen chronischen Nikotinkonsum (entsprechend 50 pack years) und einen regelmäßigen Alkoholkonsum (ca. $85 \mathrm{~g}$ tgl.) mit Zirrhosis hepatis hinzuweisen.

Hierorts präsentierte sich die Patientin in einem reduzierten Allgemeinzustand (Body-Mass-Index von $23,6 \mathrm{~kg} / \mathrm{m}^{2}$ ), fieberfrei, über der Lunge ein gedämpfter Klopfschall links basal sowie diffus obstruktive Rasselgeräusche. Der Blutdruck lag bei 135/70 mmHg, die Herzfrequenz bei 80 Schlägen/min. In der arteriellen Blutgasanalyse zeigte sich eine ausgeprägte Oxygenierungsstörung trotz Sauerstoffgabe von $5 \mathrm{LO} 2 / \mathrm{min}$ via Nasenbrille (paO2 $56 \mathrm{mmHg}$, paCO2 $47 \mathrm{mmHg}, \mathrm{pH} 7,44)$. Laborchemisch fanden sich erhöhte serologische Entzündungswerte (CRP $108 \mathrm{mg} / \mathrm{l}$, Leukozyten 31,1G/1); die Leberparameter bis auf eine diskret erhöhte $\gamma$-GT bei erhaltener Synthesefunktion im Normbereich. Radiologisch zeigte sich im Aufnahmeröntgen ein Fluidopneumothorax links, darüber hinaus bestand der Verdacht einer abszedierenden Pneumonie ( $\bullet$ Abb.1). Neben herkömmlichen therapeutischen Maßnahmen einer antiobstruktiven Therapie (inklusive systemischer Kortisongabe und inhalativer bronchodilatatorischer Therapie), erhielt die Patientin eine parenterale Antibiotikatherapie mit Piperacillin/Tazobactam. Aufgrund des nachgewiesenen LuftFlüssigkeitsspiegels wurde weiter eine konventionelle thorakale Drainage (Ch 24) in Lokalanästhesie gesetzt. Hierbei entleerte sich reichlich parapneumonisches Exsudat (initial $400 \mathrm{ml}$, in Folge zW. $150-200 \mathrm{ml} /$ Tag), die mikrobiologische Aufarbeitung erbrachte nach bereits stattgehabter Antibiotikatherapie keinen Keimnachweis. Die chemische Analyse des Aspirats zeigte einen pH-Wert von 7,25, ein LDH von 180U/L, bei einer Eiweißkonzen-

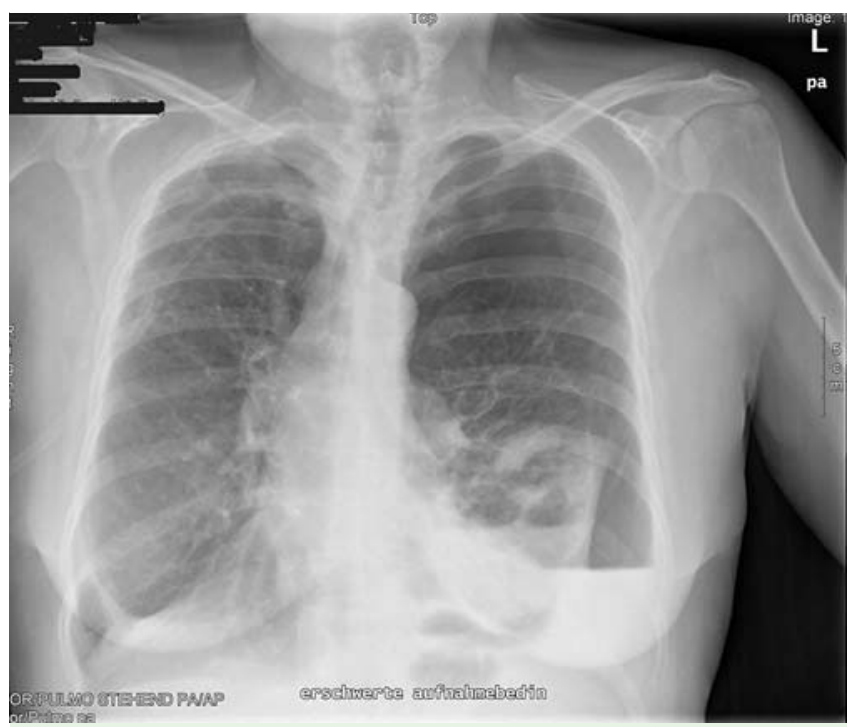

Abb. 1 Thoraxröntgen bei Aufnahme mit dem Nachweis eines Fluidopneumothorax.
Tab. 1 Parameter der Laborchemie und Blutgasanalyse zum Zeitpunkt der Aufnahme, kurz vor der Entlassung aus dem Krankenhaus (Tag 25) und zur Nachsorge (9 Monate).

\begin{tabular}{|llll|}
\hline & Aufnahme & Entlassung & Nachsorge \\
\hline Laborchemie & & & \\
\hline Leukozyten, G/I & 31,1 & 11,1 & 7,5 \\
\hline Hämoglobin, g/dl & 11,3 & 13,1 & 13,8 \\
\hline C-reaktives Protein, mg/l & 108 & 53 & 6.0 \\
\hline Arterielle Blutgasanalyse & $+5 \mathrm{LO} 2$ & $+2 \mathrm{LO} 2$ & $+2 \mathrm{lO} 2$ \\
\hline paO2, mmHg & 56 & 64 & 78 \\
\hline paCO2, $\mathrm{mmHg}$ & 47 & 38 & 40 \\
\hline
\end{tabular}

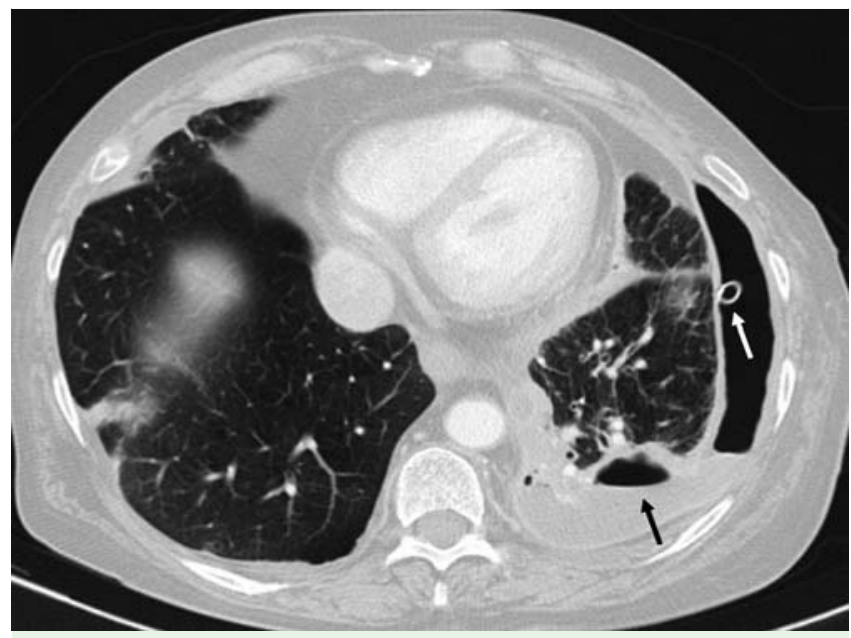

Abb.2 Abszesshöhle im linken Unterlappen mit Bronchusanschluss (schwarzer Pfeil) und liegender Thoraxdrainage (weißer Pfeil).

tration von 3,5g/dl. Unter der laufenden Therapie kam es neben einer Verbesserung des Allgemeinzustandes und der Oxygenierung zu rückläufigen serologischen Infektparametern ( Tab. 1). Trotz nativradiologisch korrekter Lage der intrathorakalen Drainage und klinischer Besserung zeigte sich jedoch noch 10 Tage nach der Intervention ein persistierendes Entfaltungsdefizit der Lunge, verbunden mit einer atemsynchronen Leckage als Ausdruck einer bronchopleuralen Fistel. Ursächlich hierfür fand sich in der darauffolgend durchgeführten Computertomografie eine BPF mit sichtbarem Bronchialanschluss in die bereits nativradiologisch suspizierte Abszesshöhle ( $\bullet$ Abb.2). Der zunächst konservative Versuch mittels Thoraxdrainage inkl. Sog $(-15 \mathrm{~cm}$ $\mathrm{H} 2 \mathrm{O}$ ) brachte keinen anhaltenden Erfolg. Eine thoraxchirurgische Sanierung der BPF war unter Berücksichtigung des erhöhten pulmonalen Risikoprofils bei fortgeschrittener COPD (FEV1 $650 \mathrm{ml}$, entspricht $27 \%$ des Solls) von der Patientin abgelehnt worden, sodass wir uns entschlossen haben, die Patientin einer bronchoskopischen Einwegventilimplantation zur Behandlung der BPF zuzuführen.

Die Untersuchung erfolgte in Allgemeinanästhesie mit Intubation via starrem Bronchoskop und Jet-Beatmung (TwinStream, Carl Reiner, Österreich). In den endobronchial einsehbaren Bronchialabschnitten fanden sich keine besonderen Auffälligkeiten. Zur Identifikation der BPF führten wir eine selektive endobronchiale Ballonblockade der einzelnen Segmentostien des linken Unterlappens durch, wobei durch Okklusion der Segmentbronchien des laterobasalen und posterobasalen Unterlappenbronchus schließlich ein Sistieren der Leckage beobachtet werden konnte. In selbiger Sitzung erfolgte sodann die Implantation von 2 endobronchialen Einwegventilen (Zephyr Ventil, 4.0, Fa. Pulmonx, 


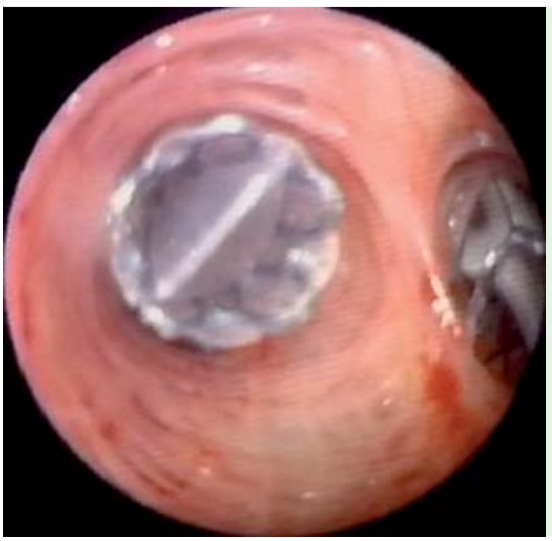

Abb.3 Endoskopische Ansicht eines endobronchialen Einwegventils zur Behandlung einer bronchopleuralen Fistel.

USA) über den Arbeitskanal des Bronchoskops ( $\bullet$ Abb.3). Die Untersuchungsdauer betrug knapp 20 Minuten; der postinterventionelle Verlauf war unauffällig und auch in weiterer Folge wurden keine Komplikationen beobachtet. Die Entfernung der Thoraxdrainage - welche sich bereits zuvor 28 Tage in situ befunden hatte - erfolgte 5 Tage nach erfolgreicher Ventilimplantation bei radiologisch gänzlich entfalteter Lunge ohne Hinweise auf eine Persistenz der BPF. Die Patientin selbst wurde schließlich am 31. Tag der stationären Betreuung in stabilem Zustand entlassen und präsentierte sich auch 9 Monate danach im Rahmen einer an unserer Abteilung durchgeführten Verlaufskontrolle, in einem anhaltend stabilen Allgemeinzustand ohne weitere Langzeitkomplikationen. In der Bildgebung mittels Thorax-CT findet sich zu diesem Zeitpunkt eine ausgedehnte Lunge mit einer Abszessresthöhle, die an Ausdehnung im Vergleich zur Voruntersuchung deutlich abgenommen hat ( Abb.4). Die endobronchialen Einwegventile wurden aufgrund weiterhin bestehender radiologischer Anzeichen einer bronchopleuralen Verbindung zunächst belassen, es wurden bis dato keine Komplikationen im Sinne relevanter Fremdkörperreaktionen beobachtet.

\section{Diskussion und Zusammenfassung \\ $\nabla$}

Im Wesentlichen unterscheiden wir heute zwischen einer spontanen und iatrogenen (postinterventionellen oder postoperativen) bronchopleuralen Fistel. Zu den häufigsten Ursachen einer spontanen BPF zählen die abszedierende Pneumonie, die Lungentuberkulose sowie der Spontanpneumothorax, vor allem bei Patienten mit Lungenemphysem [1,5,7]. Begünstigend für das Auftreten einer BPF sind eine maligne Grunderkrankung, ein Zustand nach Radiotherapie und/oder Chemotherapie sowie ein schlechter Allgemeinzustand mit Malnutrition und Kachexie [4-8]. Darüber hinaus scheint auch eine Kortikosteroidtherapie zu den relevanten krankheitsbegünstigenden Faktoren zu zählen $[5,8,11]$. Die Diagnose der BPF wird in der Regel klinisch gestellt, der Nachweis einer atemsynchronen Leckage über eine intrathorakale Drainage ist beweisend. Der zunehmend häufiger werdende Einsatz der Computertomografie ermöglicht die zugrundeliegende Ursache der BPF zu identifizieren, in Einzelfällen ist dadurch auch die segmentale Zuordnung der BPF möglich. In der klinischen Routine wird die Lokalisation der BPF jedoch via Bronchoskopie eruiert, wobei hier klassischerweise die Methylenblauinstillation oder Kontrastmittel-Bronchografie Anwendung findet $[5,6]$. Die an unserer Abteilung etablierte Technik bedient sich der endobronchialen Ballonblockade der zur Leckage führenden Bronchialabschnitte. Neben selektiver Identifikation der

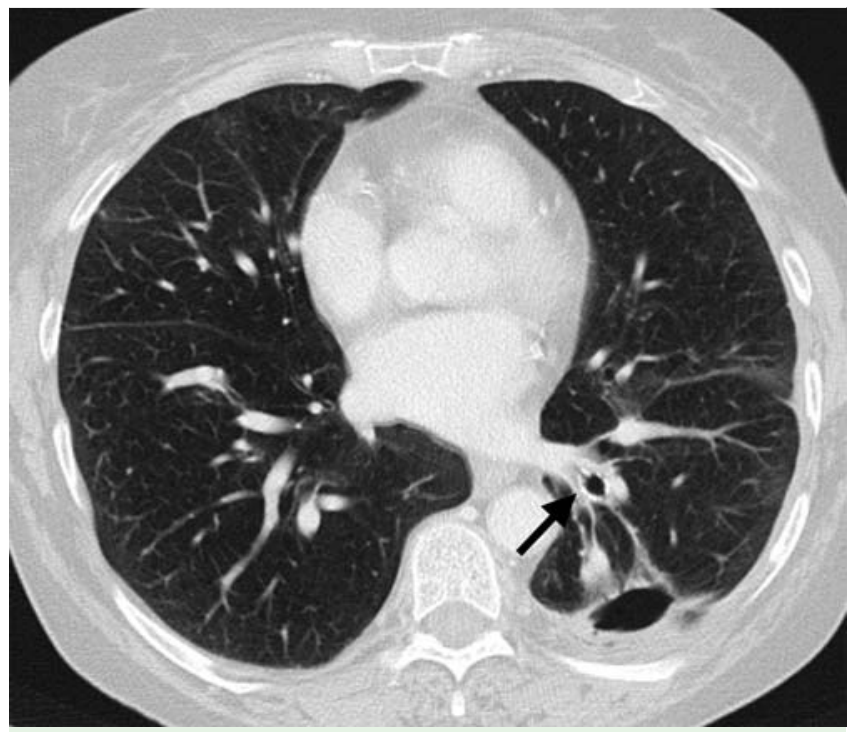

Abb.4 Thorax-Computertomografie 9 Monate nach der Intervention: Endobronchiales Einwegventil im laterobasalen linken Unterlappensegment (siehe Pfeil).

BPF ist damit in gleicher Sitzung auch eine Vorhersage möglich, ob eine endobronchiale Okklusion zielführend sein kann.

Die Behandlung der spontanen bronchopleuralen Fistel erfordert neben der Optimierung patientenbezogener Faktoren und suffizienter Antibiose in der Regel die Platzierung einer intrathorakalen Drainage. Kann dadurch keine „spontane“ Remission erzielt werden, muss somit in Fällen einer persistierenden BPF eine gezielte - meist operativ durchgeführte - Sanierung der BPF mittels Thorakotomie oder Thorakoskopie erfolgen. Bei Patienten mit erhöhtem Operationsrisiko - wie in unserer Kasuistik - ist ein primär chirurgisches Vorgehen allerdings oft nicht möglich. In solchen Fällen sind in interdisziplinärer Zusammenarbeit individualisierte, minimal-invasive, gegebenenfalls multimodale Behandlungsoptionen anzustreben. In diesem Zusammenhang wurden in der Literatur mehrere endoskopische Verfahren beschrieben, die mittels endobronchialer Okklusion die Ventilation zur Parenchymleckage zu blockieren versuchen, um dadurch schließlich eine Spontanheilung zu ermöglichen.

Unter anderem wurde anhand von Fallbeispielen der Einsatz von Fibrinkleber [12], Gelschaum [13], die Instillation von Albuminkomplex [14] oder Ethanol beschrieben [15]. Die Erfolgsrate der oben angeführten Maßnahmen ist jedoch anhand der geringen Fallzahlen und fehlenden systematischen und vergleichenden Studien nur schwer vorhersagbar bzw. auf ein großes und heterogenes Patientenkollektiv schwer übertragbar. Ausschlaggebend ist als wesentliches Entscheidungskriterium, neben der Lokalisation und Größe der BPF, vor allem auch die Erfahrung und Routine des Untersuchers mit den verschiedenen Methoden. Als mögliche Alternative zu den oben angeführten Okklusionstechniken wurde kürzlich eine Fallserie von Patienten mit BPF beschrieben, bei denen mittels Platzierung des AmplatzerSchirmchens [16-18] ein Verschluss derselbigen erreicht werden konnte. Vor allem bei größeren Defekten $(>8 \mathrm{~mm})$ scheint dies eine interessante Option zu sein.

Die in unserem Fallbeispiel benutzte Methode bedient sich der Implantation eines endobronchialen Einwegventils, welches auch im Rahmen der bronchoskopischen Lungenvolumsreduktion bei Emphysem Anwendung findet $[4,7,8]$. Durch den unidirektionalen Block der Ventilation wird einerseits ein weiterer 
Austritt von Luft in den Pleuraraum verhindert, gleichzeitig bleibt jedoch der Sekretabfluss über das Bronchialsystem bzw. den Atemweg erhalten. Dadurch wird die Spontanheilung des Pleuradefektes - und somit der Verschluss der BPF - unterstützt, was letztlich einen wesentlichen Benefit dieser Methode gegenüber den meisten oben angeführten Okklusionstechniken ausmacht. Weitere Vorteile der Ventilimplantation liegen neben der relativ einfachen Handhabung vor allem in der niedrigen peri- und postinterventionellen Komplikationsrate hinsichtlich Fremdgewebsreaktion, Infektion und Dislokation. Letztere kann durch radiologische Verlaufskontrollen und Nachweis der Ventilposition erkannt werden. Darüber hinaus handelt es sich bei diesem Vorgehen um ein potenziell reversibles Verfahren, nach entsprechender Rekonvaleszenz ist somit die Entfernung des Ventils jederzeit möglich.

Die ersten Berichte über Verwendung dieser Technik zur Sanierung einer BPF stammen aus dem Jahr 2005. Snell et al. [19] berichteten erstmalig über den Einsatz von Einwegventilen in dieser Indikation bei einem Pat., der, nach mehrfachen operativen Eingriffen aufgrund eines Aspergilloms und daraus resultierender BPF, nach 6-jährigem Leidensweg schließlich erfolgreich saniert werden konnte. Es wurden dabei in Allgemeinanästhesie mehrere endobronchiale Einweg-Ventile fiberoptisch platziert; die Intervention nahm 50 Minuten in Anspruch, und der Pat. konnte nach unauffälligem Verlauf noch am selben Tag entlassen werden und zeigte sich 4 Monate nach dem Eingriff in einem deutlich gebesserten Allgemeinzustand.

Ferguson et al. [7] konnten mittels bronchoskopischer Ballonblockade bei einer 63-jährigen Tumorpatientin als Ursache eines postoperativen persist. Fluidopneumothorax ( $>9$ Tage) eine BPF nachweisen und diese ebenfalls mittels fiberoptisch platziertem Ventil beheben. Die Entfernung der Thoraxdrainagen war einen Tag später möglich, und auch 4 Monate postinterventionell war kein neuerliches Pneumothoraxrezidiv aufgetreten. Das Ventil wurde auch in diesem Fallbeispiel in situ belassen.

Rezent wurde auch ein größeres Patientenkollektiv im Rahmen einer retrospektiven, nicht randomisierten Studie untersucht. Travaline et al. [8] untersuchten 40 Patienten mit einer BPF unterschiedlichster Ätiologie, die mit endobronchialen Ventilen (ø 20 Tage nach Diagnose der BPF) versorgt wurden, wobei in 6 Fällen Komplikationen (unter anderem Ventilaushusten, moderate Sauerstoffdesaturation, Fehlpositionierung mit Notwendigkeit zur Rebronchoskopie sowie Pneumonie) auftraten; ein komplettes Sistieren der Leckage war bei 19 Pat. bzw. eine Reduktion in 18 Fällen dokumentierbar; bei 2 Pat. kam es zu keiner Besserung der BPF. Die Thoraxdrainagen konnten durchschnittlich 7,5 Tage nach Ventilimplantation entfernt werden; der mittlere Krankenhausaufenthalt nach Implantation lag bei 11 Tagen. Im Rahmen der Nachbeobachtungsphase konnten keine weiteren Komplikationen bzw. Todesfälle in Zusammenhang mit den Ventilen gefunden werden [8]. Die Entfernung der Thoraxdrainage als auch die Entlassung unserer Pat. aus dem stationären Aufenthalt liegt im durchschnittlichen Zeitrahmen der o.g. Studie.

Der Einsatz dieser Methode ist auf Basis bisheriger Erfahrungen bei Patienten mit persistierenden BPF in Erwägung zu ziehen. Wenn man die Literatur vergleicht, bietet die endobronchiale Ventilimplantation vor allem jenen Patienten eine minimal-invasive Alternative, die sich in einem schlechten Performancestatus befinden und deren Komorbiditäten keine operative Sanierung erlauben. Der Eingriff kann sowohl in Allgemein- als auch Lokalanästhesie durchgeführt werden, darüber hinaus ist die Implantation der Ventile an sich mit wenigen Komplikationen behaftet und potenziell reversibel.
Zusammenfassend konnten wir einer Patientin mit fortgeschrittener COPD und Lungenabszess durch die Implantation endobronchialer Einwegventile eine minimal-invasive und erfolgreiche Alternative in der Behandlung einer bronchopleuralen Fistel anbieten. Aufgrund mangelnder Vergleichsstudien bleibt derzeit die Wahl aus den zur Verfügung stehenden Behandlungsmöglichkeiten eine individuelle Entscheidung, wobei neben patientenbezogenen Faktoren vor allem der Erfahrung des Interventionisten eine tragende Rolle zukommt.

\section{Interessenkonflikt}

Doz. Dr. A. Valipour war lokaler Studienleiter im Rahmen der VENT-Studie. Darüber hinaus ist Doz. Dr. Valipour in der Vergangenheit als Konsulent für die Fa. Pulmonx tätig gewesen. Die anderen Autoren haben keine Interessenskonflikte.

\section{Literatur}

1 Baumann MH, Sahn SA. Medical Management and therapy of bronchopleural fistulas in the mechanically ventilated patient. Chest 1990; 97: $721-728$

2 Sirbu H, Busch T, Aleksic I et al. Bronchopleural fistula in the surgery of non-small lung cancer: Incidence, Risk Factors, and Management. Ann Thorac Cardiovasc Surg 2001; 7: 330-336

3 Lin J, Iannettoni MD. Closure of bronchopleural fistulas using albuminglutaraldehyde tissue adhesive. Ann Thorac Surg 2004; 77: 326-328

4 Feller-Kopmann D, Bechara R, Garland R et al. Use of Removable Endobronchial Valve for the Treatment of Bronchopleural Fistula. Chest 2006; 130: $273-275$

5 Lois $M$, Noppen $M$. Bronchopleural fistulas: an overview of the problem with special focus on endoscopic management. Chest 2005; 128: $3955-3965$

6 McManigle JE, Fletcher GL, Tenholder MF. Bronchoscopy in the Management of Bronchopleural Fistula. Chest 1990; 97: 1235-1238

7 Ferguson JS, Sprenger K, Van Natta T. Closure of a bronchopleural fistula using bronchoscopic placement of an endobronchial valve designed for the treatment of emphysema. Chest 2006; 129: 479-481

8 Travaline JM, McKenna RJ, De Giacomo T et al. Treatment of persistent pulmonary air leaks using endobronchial valves. Chest 2009; 136: $355-360$

9 Koh MS, Ling Hsu AA, Thirugnanam A. Novel Management of a large chronic bronchocutaneous fistula after lobectomy. Interact Cardio Vasc Thorac Surg 2005; 4: 248-249

10 Sarkar P, Chandak T, Shah $R$ et al. Diagnosis and management bronchopleural fistula. Indian J Chest Dis Allied Sci 2010; 52: 97-104

11 Asamura $H$, Kondo H, Goya $T$ et al. Bronchopleural fistulas developing after pulmonary resections for lung cancer predisposing factors, management, and prognosis (Article in Japanese). Nippon Kyobu Geka Gakkai Zasshi 1991; 39: 1894-1901

12 Petter-Puchner AH, Simunek M, Redl H et al. A comparison of a cyanoacrylate [corrected] glue (Glubran) vs. fibrin sealant (Tisseel) in experimental models of partial pulmonary resection and lung incision [corrected] in rabbits. J Invest Surg 2010; 23: 40-47

13 Jones DP, David I. Gelfoam occlusion of peripheral bronchopleural fistulas. Ann Thorac Surg 1986; 42: 334-335

14 Andreetti C, D'Andrilli A, Ibrahim $M$ et al. Submucosal injection of the silver-human albumin complex for the treatment of bronchopleural fistula. Eur J Cardiothorac Surg 2010; 37: 40-43

15 Takaoka K, Inoue S, Ohira S. Central bronchopleural fistulas closed by bronchoscopic injection of absolute ethanol. Chest 2002; 122: 374-378

16 Gulkarov I, Paul S, Altorki NK et al. Use of Amplatzer device for endobronchial closure of bronchopleural fistulas. Interact Cardiovasc Thorac Surg 2009; 9: 901 - 902

17 Scordamaglio PR, Tedde ML, Minamoto $H$ et al. Endoscopic treatment of tracheobronchial tree fistulas using atrial septal defect occluders: preliminary results. J Bras Pneumol 2009; 35: 1156-1160

18 Kramer MR, Peled N, Shitrit D et al. Use of Amplatzer device for endobronchial closure of bronchopleural fistulas. Chest 2008; 133: 1481 1484

19 Snell GI, Holsworth L, Fowler S et al. Occlusion of a broncho-cutaneous fistula with endobronchial one-way valves. Ann Thorac Surg 2005; 80: $1930-1932$ 